\title{
Denosumab Versus Zoledronic Acid in Bone Disease Treatment of Newly Diagnosed Multiple Myeloma: An International, Double-Blind, Randomized Controlled Phase 3 Study-Asian Subgroup Analysis
}

\author{
Shang-Yi Huang · Sung-Soo Yoon - Kazuyuki Shimizu - Wee Joo Chng · \\ Cheng-Shyong Chang · Raymond Siu-Ming Wong · Seasea Gao • \\ Yang Wang · Steve W. Gordon · Anthony Glennane · Chang-Ki Min
}

Received: April 29, 2020 / Published online: June 10, 2020

(C) The Author(s) 2020

\section{ABSTRACT}

Introduction: The primary analysis of a global phase 3 study that evaluated the efficacy and safety of denosumab versus zoledronic acid for preventing skeletal-related events (SREs) in adults with newly diagnosed multiple myeloma (MM) indicated that denosumab was noninferior to zoledronic acid for time to first on-study

Digital Features To view digital features for this article go to https://doi.org/10.6084/m9.figshare.12349892.

S.-Y. Huang

Division of Hematology, Department of Internal

Medicine, National Taiwan University Hospital,

Taipei, Taiwan

\section{S.-S. Yoon}

Department of Internal Medicine, Seoul National

University Hospital, Seoul, Republic of Korea

K. Shimizu

Department of Hematology/Oncology, Higashi

Nagoya National Hospital, Nagoya, Japan

\section{W. J. Chng}

Department of Hematology-Oncology, National

University Cancer Institute, Singapore, National

University Health System, Singapore,

Republic of Singapore

\section{W. J. Chng}

Cancer Science Institute of Singapore, National

University of Singapore, Singapore,

Republic of Singapore
SREs. Here we present a subgroup analysis to evaluate efficacy and safety in Asian patients.

Methods: Patients were randomized 1:1 to receive denosumab $120 \mathrm{mg}$ subcutaneously or zoledronic acid intravenously $4 \mathrm{mg}$ every 4 weeks in a double-blind, double-dummy fashion. All patients received standard-of-care first-line antimyeloma treatment. Each patient received either study drug until an estimated 676 patients experienced at least one on-study SRE and the primary efficacy and safety analyses were completed.

\section{W. J. Chng}

Department of Medicine, Yong Loo Lin School of

Medicine, Singapore, Republic of Singapore

\section{C.-S. Chang}

Department of Internal Medicine, Chang Bing Show Chwan Memorial Hospital, Changhua, Taiwan

R. S.-M. Wong

Sir Y.K. Pao Centre for Cancer and Department of Medicine and Therapeutics, The Chinese University of Hong Kong, Hong Kong SAR, China

S. Gao $\cdot$ S. W. Gordon

Amgen Asia Holding Ltd., Hong Kong SAR, China

Y. Wang · A. Glennane

Amgen Inc., Thousand Oaks, CA, USA

C.-K. Min $(\bowtie)$

Department of Hematology, Seoul St. Mary's

Hospital, Seoul, Republic of Korea

e-mail: ckmin@catholic.ac.kr 
Results: Of 1718 total enrolled patients, 196 Asian patients (denosumab, $n=103$; zoledronic acid, $n=93$ ) were included in this subgroup analysis. Fewer patients in the denosumab group developed first on-study SRE compared with the zoledronic acid group; the crude incidence of SREs at the primary analysis cutoff was $38.8 \%$ and $50.5 \%$, respectively (HR [95\% CI], 0.77 [0.48-1.26]). All 194 patients receiving at least one dose of study drug experienced at least one treatment-emergent AE. The most common AEs reported in either group (denosumab, zoledronic acid) were diarrhea (51.0\%, 51.1\%), nausea $(42.2 \%, 46.7 \%)$, and pyrexia $(38.2 \%$, $41.3 \%)$. Treatment-emergent renal toxicity occurred in 9/102 (8.8\%) and 20/92 (21.7\%) patients, respectively. Similar rates of positively adjudicated osteonecrosis of the jaw (7 [6.9\%] vs 5 [5.4\%]) and treatment-emergent hypocalcemia (19 [18.6\%] vs 17 [18.5\%]) were reported in the denosumab and zoledronic acid groups, respectively.

Conclusion: Efficacy and safety outcomes from this Asian subgroup were comparable to those of the full study population. Overall, this analysis supports denosumab as an additional treatment option for standard of care for Asian patients with newly diagnosed MM with lytic bone lesions.

Clinical Trial Registration: ClinicalTrials.gov NCT01345019.

Keywords: Asian patients; Denosumab; Multiple myeloma; Skeletal-related event; Zoledronic acid

\section{Key Summary Points}

Why carry out this study?

Multiple myeloma (MM) is characterized by development of osteolytic lesions, which result from deregulation of normal bone remodeling, causing cancer-induced bone loss and destruction, and increased risk for fracture
The primary analysis of a global phase 3 study (NCT01345019) indicated that denosumab was noninferior to zoledronic acid for time to skeletal-related events (SREs) in patients with newly diagnosed $\mathrm{MM}$ with at least one lytic bone lesion; here we present a subgroup analysis of this study to evaluate efficacy and safety in patients from Asian countries

What was learned from this study?

Fewer patients in the denosumab group developed first on-study SRE compared with the zoledronic acid group, results that are consistent with those from the full study

Rates of adverse events, including adjudicated osteonecrosis of the jaw and hypocalcemia, were generally similar between the two treatment groups, renal toxicity was less frequent in the denosumab than in the zoledronic acid group, and overall safety results for the Asian subgroup were in line with those from the full study

Efficacy and safety outcomes for denosumab and zoledronic acid from this Asian subgroup analysis of patients with MM were comparable to those from the primary analysis of the full study population, supporting the use of denosumab as an additional treatment option for the standard of care for Asian patients with newly diagnosed MM with osteolytic lesions

\section{INTRODUCTION}

Multiple myeloma (MM) is a plasma cell malignancy with approximately 150,000 new cases each year worldwide [1]. In 2018, approximately 56,676 new MM cases were reported in Asia, including 6313 in Japan, 1905 in Korea, 168 in Singapore, and 227 in Malaysia [2-6]. In 2016, approximately 270 new cases of MM were reported in Hong Kong [7]. From 2011 to 2012, 1023 new cases of MM were reported in Taiwan [8]. 
MM is characterized by osteolytic lesions, renal dysfunction, hypercalcemia, anemia, reduced levels of normal immunoglobulins, and increased infection risk [9]. Bone destruction is one of the devastating consequences of $\mathrm{MM}$ $[10,11]$; the severity of bone destruction correlates with tumor burden and prognosis [12]. The interaction of MM cells with the bone marrow microenvironment deregulates a number of signaling pathways, causing the increased release of factors that potentiate osteoclast formation and activation while inhibiting osteoblast differentiation, leading to greater bone resorption and the suppression of bone formation, respectively $[10,13]$. Deregulation of the receptor activator of nuclear factor-kappa $\mathrm{B}$ (RANK)/RANK ligand (RANKL) signaling pathway has been primarily linked to the pathogenic increase in osteoclast activity observed in MM [10, 13]. Additional osteoclastogenic factors implicated are the macrophage inflammatory protein $1 \alpha$ (MIP-1 $\alpha)$, interleukin-1 (IL-1), IL-3, and IL-6 [10, 13].

A study of patients with MM in China, Hong Kong, Japan, Korea, Singapore, Taiwan, and Thailand revealed that the median age at diagnosis was 62 years [14], which is lower than that reported for the USA (69 years) [15]. Asian patients as a whole are more likely to have advanced disease due to the delay in diagnosis compared to Western countries, with rates of International Staging System (ISS) stages I, II, and III of 19.9\%, $36.1 \%$, and $44.0 \%$, respectively. Overall, $60.2 \%$ of Asian patients had documented bone lesions, ranging from $28.5 \%$ of patients in Thailand to $80.0 \%$ of patients in Japan, consistent with rates reported in Western countries [14].

Intravenously administered (IV) bisphosphates, such as zoledronic acid, are considered the standard of care in the management of myeloma bone disease with demonstrated efficacy across tumor types [16-18]. However, despite the use of IV bisphosphonates, a substantial number of patients with MM develop skeletal complications [19]. In addition, bisphosphonate use is discouraged in patients with renal dysfunction [20], particularly based on the evidence that approximately $60 \%$ of patients present with renal dysfunction during the course of the disease [17]. Denosumab is a monoclonal antibody targeting RANKL that has been shown to reduce skeletal-related events (SREs) associated with bone lesions in patients with MM [21-23].

In patients with solid tumors that had metastasized to bone, denosumab $120 \mathrm{mg}$ subcutaneously administered (SC) every 4 weeks (Q4W) was superior to zoledronic acid in delaying time to SREs [11, 22, 24-27]. Subsequently, SC denosumab $120 \mathrm{mg}$ Q4W was found to be noninferior to IV zoledronic acid $4 \mathrm{mg}$ Q4W for preventing SREs in a phase 3 study in patients with newly diagnosed MM [21]. Overall survival was similar in both groups (median 49.5 months for denosumab vs not estimable for zoledronic acid; HR 0.90 [95\% CI $0.70-1.16], P=0.41)$. An exploratory endpoint of this study, progression-free survival (PFS), showed a numerical advantage for denosumab of 10.7 months, compared to zoledronic acid (median 46.1 vs 35.4 months; HR 0.82 [95\% CI 0.68-0.99], descriptive $P=0.036$ ) [21]. For Asian patients with $\mathrm{MM}$, there is no direct comparison between these two drugs in the literature. Herein, we assess the consistency of Asian patients receiving denosumab or zoledronic acid with the full study population.

\section{METHODS}

Methods for the primary analysis (NCT01345019) [21] of this study were previously described in detail and are briefly summarized here. The Asian subgroup used for this analysis included patients enrolled from the Republic of Korea, Japan, Taiwan, Singapore, Malaysia, and Hong Kong.

\section{Study Design and Participants}

This study was a multicenter, randomized, double-blind, active-controlled, phase 3 trial in adults with newly diagnosed MM. Patients were enrolled if they were adults aged at least 18 years with newly diagnosed MM, at least one lytic bone lesion, an Eastern Cooperative Oncology Group (ECOG) score of $0-2$, adequate hepatic function (i.e., tests of hepatic function at most two times the upper limit of normal) 


\section{BLINDED TREATMENT PHASE}

\section{OPEN-LABEL PHASE}

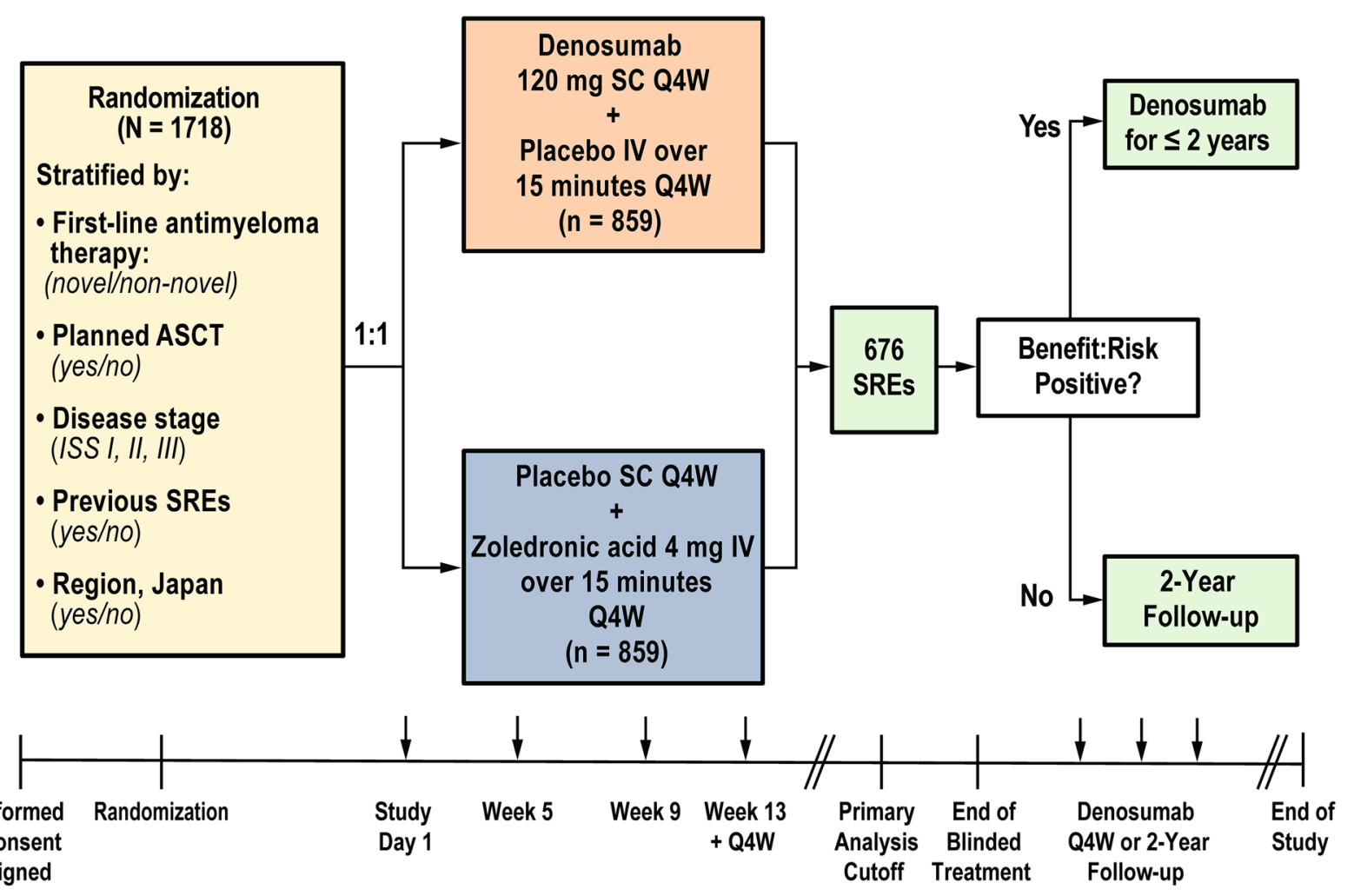

Fig. 1 Study design and treatment schema. ASCT autologous stem cell transplant, ISS International Staging System, $I V$ intravenously administered, $Q 4 W$ every 4 weeks, $S C$ subcutaneously administered, $S R E$ skeletal-related event

and adequate renal function (i.e., creatinine clearance of at least $30 \mathrm{~mL} / \mathrm{min})$. Key exclusion criteria included having plasma cell leukemia, receipt of more than 30 days of antimyeloma therapy before screening, receipt of more than one previous dose of IV bisphosphonate, and non-healed dental or oral surgery.

Patients were randomized 1:1 to receive SC denosumab $120 \mathrm{mg}$ Q4W plus IV placebo or IV zoledronic acid $4 \mathrm{mg}$ Q4W plus SC placebo (Fig. 1). All patients also received investigators' choice of first-line antimyeloma therapy, and supplementation with daily vitamin D and calcium was strongly encouraged, unless the patient had documented hypercalcemia. Denosumab dose adjustments were not permitted; zoledronic acid dose adjustments followed approved prescribing guidelines.

Randomization was blinded and stratified by antimyeloma therapy (novel vs non-novel therapy), intent to undergo autologous stem cell transplantation (yes vs no), ISS stage at diagnosis (I, II, or III), previous SREs (yes vs no), and region (Japan vs other). Blinded treatment continued until approximately 676 patients had at least one on-study SRE (i.e., primary analysis cutoff) and the primary efficacy and safety analysis was completed. Subsequently, open-label denosumab was offered to each patient for up to 2 years.

The study was conducted in accordance with Good Clinical Practice, and study investigators obtained approval from their respective independent ethics committee or institutional review board before study initiation. Written informed consent was provided by patients before any protocol-specific procedures and before the administration of any study drug.

\section{Outcomes and Assessments}

The primary endpoint for the primary analysis was whether denosumab was noninferior to 
zoledronic acid with regards to time to first onstudy SRE (noninferiority), defined as the time in days from the date of randomization to the date of the first occurrence of an on-study SRE. If there was no SRE, then time to first on-study SRE was censored at the date the patient completed the treatment phase or the primary analysis cutoff date, whichever was earlier. The effect of study treatment on PFS (time in days from randomization date to the date of first recorded overall disease progression [as determined by study investigators] or to death during the treatment phase from any cause, whichever came first) was assessed as an exploratory endpoint.

In this subgroup analysis, the time to first on-study SRE (tests of noninferiority were not undertaken) and PFS were estimated. The safety and tolerability of denosumab compared with zoledronic acid were assessed. Treatment-emergent adverse events (AEs) were defined as any untoward event occurring from the time of the first dose of study drug through to 30 days after the last dose of study drug or the end-of-treatment phase visit, whichever was longer.

Additional assessments included determining the number of months in the study per treatment group (time period from the first dose of study drug, or randomization date if patients did not receive a treatment dose, to the end of study date or primary data cutoff date, whichever came first) and the cumulative study drug exposure (days from the first dose to the last dose of study drug plus 28 days).

\section{Statistical Analyses}

This Asian subgroup analysis is descriptive in nature. Time to first on-study SRE and PFS were estimated using the Kaplan-Meier method and analyzed using a Cox proportional-hazards model stratified by randomization factors; in addition for PFS, the model was adjusted for baseline covariates (i.e., age, risk per cytogenetic-based prognosis, creatinine clearance [ $\leq 60 \mathrm{~mL} / \mathrm{min}$ vs $>60 \mathrm{~mL} / \mathrm{min}]$, ECOG $[\leq 1$ vs 2]). Incidence of treatment-emergent AEs and AEs of interest were summarized by treatment group. Time to first on-study SRE and PFS analyses in the Asian subgroup were conducted in the full analysis set, which includes all Asian patients randomized to the study. All safety analyses in the Asian subgroup were performed using randomized Asian patients who received at least one dose of study drug. Analyses were done with SAS version 9.4 and based on the data collected through the primary analysis data cutoff date of July 19, 2016.

\section{RESULTS}

\section{Patients}

The principal analysis included 1718 patients from 259 centers across 29 countries [21]. Among the entire cohort, a total of 196 (11.4\% of the total population) Asian patients were included in the subgroup analysis, including 84 (43\%) from Korea, 42 (21\%) from Japan, 26 (13\%) from Taiwan, 21 (11\%) from Singapore, 14 (7\%) from Malaysia, and 9 (5\%) from Hong Kong. Patient demographics and baseline disease characteristics were generally well balanced between treatment groups in the Asian subgroup, although there was a higher percentage of men in the denosumab group $(63.1 \%$ vs $46.2 \%$ in the zoledronic acid group) and slightly more patients treated with denosumab had an ECOG score of 2 and were diagnosed as ISS stage III at baseline compared to patients treated with zoledronic acid. Additionally, history of SRE prior to study enrollment was higher in patients in the zoledronic acid group compared to those in the denosumab group (Table 1).

The median (interquartile range [IQR]) number of months in the study was slightly shorter for patients in the denosumab group (17.5 [9.8-30.2] months) than those in the zoledronic acid group (20.2 [13.1-29.2] months). Median (IQR) cumulative drug exposure was accordingly slightly lower for patients treated with denosumab (15.9 [8.5-24.0] months) than zoledronic acid (17.4 [9.1-26.7] months). 
Table 1 Baseline demographics and disease characteristics of Asian subgroups

\begin{tabular}{|c|c|c|}
\hline & $\begin{array}{l}\text { Denosumab } 120 \mathrm{mg} \mathrm{SC} \mathrm{Q} 4 \mathrm{~W} \\
(N=103)^{\mathrm{a}}\end{array}$ & $\begin{array}{l}\text { Zoledronic acid } 4 \text { mg IV Q4W } \\
(N=93)^{\mathbf{b}}\end{array}$ \\
\hline \multicolumn{3}{|l|}{ Sex, $n(\%)$} \\
\hline Men & $65(63.1)$ & $43(46.2)$ \\
\hline Women & $38(36.9)$ & $50(53.8)$ \\
\hline Age, median (Q1, Q3) & $61.0(54.0,69.0)$ & $61.0(54.0,68.0)$ \\
\hline \multicolumn{3}{|l|}{ ECOG performance status at study entry, $n(\%)$} \\
\hline 0 & $33(32.0)$ & $24(25.8)$ \\
\hline 1 & $45(43.7)$ & $52(55.9)$ \\
\hline 2 & $25(24.3)$ & $17(18.3)$ \\
\hline \multicolumn{3}{|l|}{ Multiple myeloma ISS stage at diagnosis, $n(\%)$} \\
\hline I & $25(24.3)$ & $31(33.3)$ \\
\hline II & $35(34.0)$ & $36(38.7)$ \\
\hline III & $42(40.8)$ & $26(28.0)$ \\
\hline Not available & $1(1.0)$ & 0 \\
\hline \multicolumn{3}{|l|}{ History of SREs, $n$ (\%) } \\
\hline Any SRE & $64(62.1)$ & $70(75.3)$ \\
\hline Pathological fracture & $57(55.3)$ & $60(64.5)$ \\
\hline Spinal cord compression & $14(13.6)$ & $16(17.2)$ \\
\hline Radiation therapy to bone & $6(5.8)$ & $8(8.6)$ \\
\hline Surgery to bone & $9(8.7)$ & $22(23.7)$ \\
\hline $\begin{array}{l}\text { Prior radiotherapy to soft tissue/mass for multiple } \\
\text { myeloma, } n(\%)\end{array}$ & $2(1.9)$ & $6(6.5)$ \\
\hline Prior oral bisphosphonate use, $n(\%)$ & $1(1.0)$ & $4(4.3)$ \\
\hline \multicolumn{3}{|l|}{ Class of the first-line therapy, $n(\%)$} \\
\hline PI only & $37(35.9)$ & $42(45.2)$ \\
\hline IMiD only & $26(25.2)$ & $31(33.3)$ \\
\hline $\mathrm{PI}+\mathrm{IMiD}$ & $32(31.1)$ & $17(18.3)$ \\
\hline Other & $7(6.8)$ & $3(3.2)$ \\
\hline \multicolumn{3}{|l|}{ Cytogenetics risk group, $n$ (\%) } \\
\hline Standard risk & $77(74.8)$ & $71(76.3)$ \\
\hline High risk & $10(9.7)$ & $9(9.7)$ \\
\hline
\end{tabular}


Table 1 continued

\begin{tabular}{lll}
\hline & $\begin{array}{l}\text { Denosumab 120 mg SC Q4W } \\
(\boldsymbol{N}=103)^{\mathbf{a}}\end{array}$ & $\begin{array}{l}\text { Zoledronic acid 4 mg IV Q4W } \\
(\boldsymbol{N}=\mathbf{9 3})^{\mathbf{b}}\end{array}$ \\
\hline Unknown & $16(15.5)$ & $13(14.0)$ \\
\hline
\end{tabular}

ECOG Eastern Cooperative Oncology Group, IMiD immunomodulatory drug, ISS International Staging System, $I V$ intravenously, $P I$ proteasome inhibitor, $Q 4 W$ every 4 weeks, $S C$ subcutaneously, SRE skeletal-related event

${ }^{a}$ Of the 103 patients randomized to denosumab, 36 (35\%) were from Korea, 24 (23\%) were from Japan, 15 (15\%) were from Taiwan, 15 (15\%) were from Singapore, 8 (8\%) were from Malaysia, and $5(5 \%)$ were from Hong Kong

b Of the 93 patients randomized to zoledronic acid, 48 (52\%) were from Korea, 18 (19\%) were from Japan, 11 (12\%) were from Taiwan, 6 (6\%) were from Singapore, 6 (6\%) were from Malaysia, and 4 (4\%) were from Hong Kong

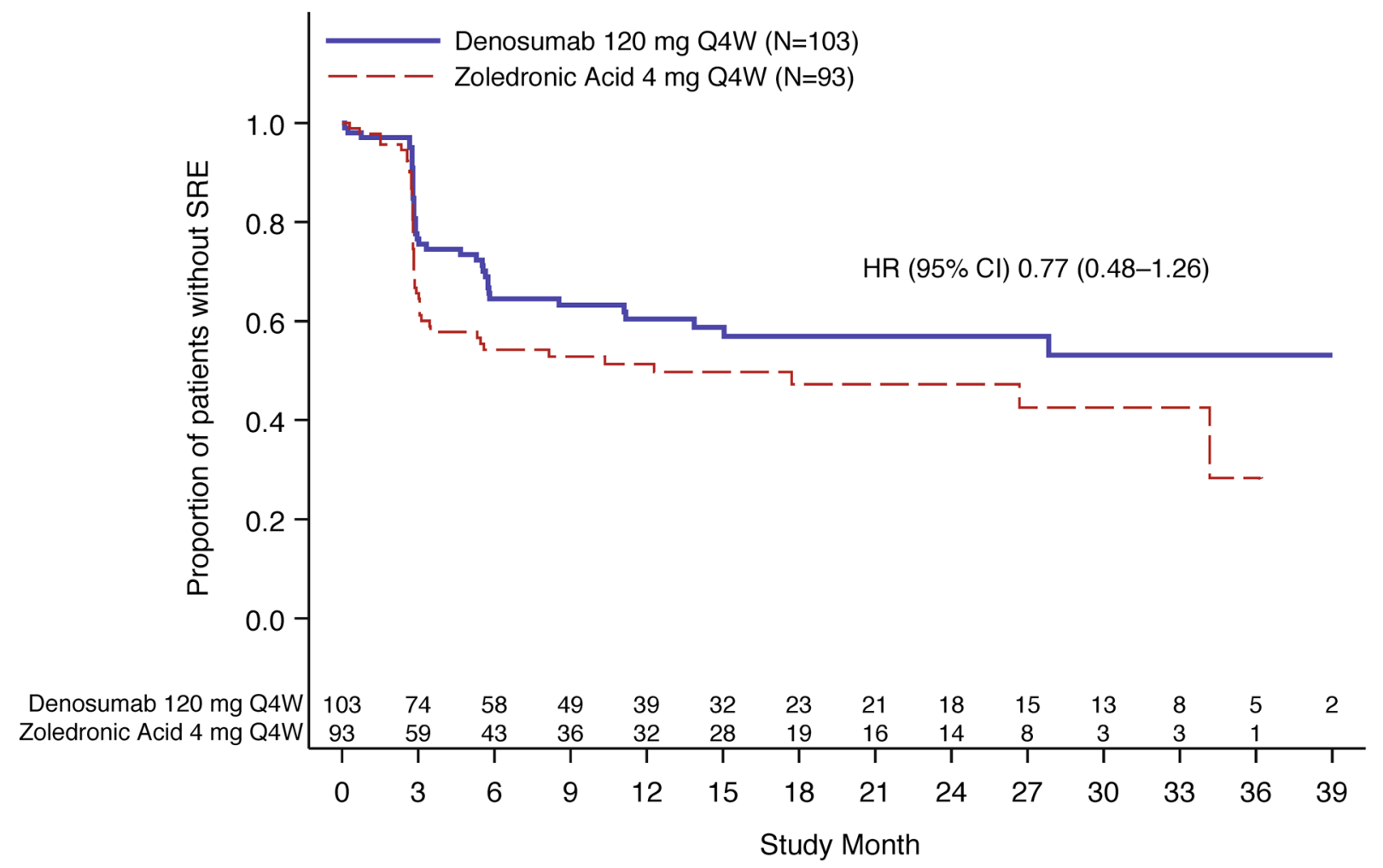

Fig. 2 Time to first on-study SRE for Asian subgroup. $C I$ confidence interval, $H R$ hazard ratio, $N$ number of patients randomized in Asian subgroup, $Q 4 W$ every 4 weeks, SRE skeletal-related event. HR $<1$ favors denosumab

\section{Outcomes}

\section{Time to First On-Study SRE}

Fewer Asian patients in the denosumab group developed a first on-study SRE compared with the zoledronic acid group (Fig. 2). The crude incidence of SREs was $38.8 \%$ in the denosumab group compared with $50.5 \%$ in the zoledronic $\left.\begin{array}{lllll}\text { acid group (hazard ratio } & {[95 \%} & \mathrm{CI}\end{array}\right]$
[0.48-1.26]). The probability (95\% CI) of patients with SRE at each time point evaluated was numerically lower for patients in the denosumab group versus those in the zoledronic acid group: at the 25th week, 29.9\% (21.8-40.2) versus $44.6 \%$ (35.0-55.4), respectively; at the 49th week, $36.8 \%(27.9-47.4)$ versus $48.7 \%$ (38.7-59.7), respectively; at the 
109th week, $43.1 \%(33.3-54.3)$ versus $52.8 \%$ (42.1-64.3), respectively.

\section{Progression-Free Survival}

A PFS event had occurred in 63 Asian patients overall (32.1\%), including 31/103 (30.1\%) patients in the denosumab group and $32 / 93$ (34.4\%) patients in the zoledronic acid group. The median PFS (95\% CI) was 29.7 (23.2-NE) months in the denosumab group and 30.2 (24.6-NE) months in the zoledronic acid group (hazard ratio $[95 \% \mathrm{CI}], 0.71$ [0.39-1.28]; descriptive $P=0.26$ ) (Fig. 3).

\section{Safety and Tolerability}

All Asian patients who received at least one active dose of denosumab or zoledronic acid experienced at least one treatment-emergent AE. The most common AEs reported in either group were diarrhea, nausea, pyrexia, upper respiratory tract infection, and constipation
(Table 2). Fewer treatment-emergent AEs associated with renal toxicity occurred in the Asian patients receiving denosumab $(8.8 \%$ [9/102]) versus zoledronic acid (21.7\% [20/92]; Table 3). The most common AE associated with renal toxicity was an increased level of serum creatinine.

For AEs of interest, the incidences of positively adjudicated osteonecrosis of the jaw were $6.9 \%(7 / 102)$ and $5.4 \%(5 / 92)$ in the denosumab and zoledronic acid groups, respectively, and the incidences of treatment-emergent hypocalcemia AEs were 18.6\% (19/102) and 18.5\% (17/ 92), respectively. Other AEs of interest (e.g., musculoskeletal pain, cardiac disorders, vascular disorders, and infections) also occurred in similar percentages of Asian patients in each treatment group (data not shown). Treatmentemergent AEs potentially associated with hypersensitivity were reported in $43.1 \%$ (44/ $102)$ versus $35.9 \%(33 / 92)$ of patients in the denosumab and zoledronic acid groups,

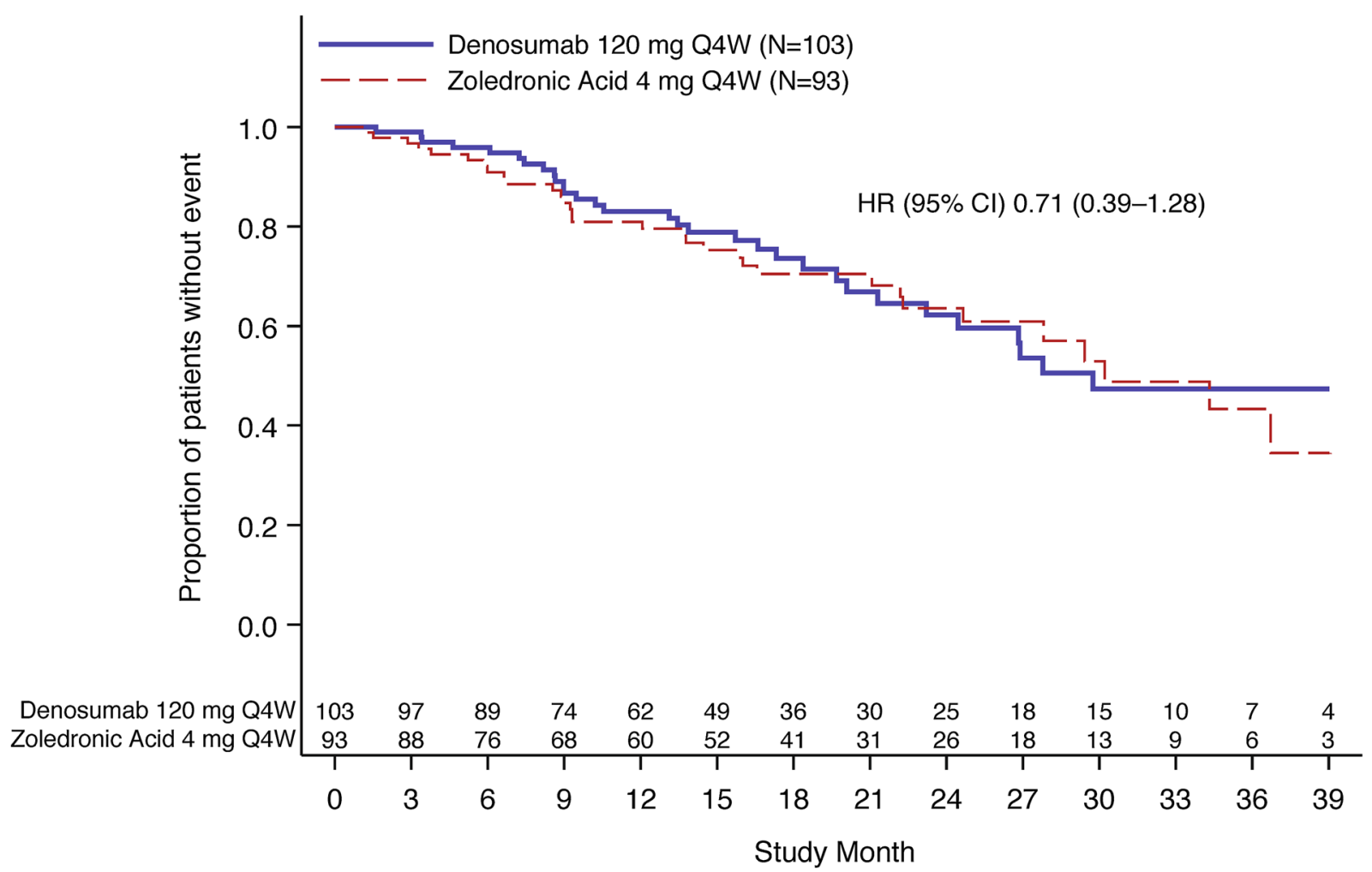

Fig. 3 Progression-free survival for Asian subgroup. $C I$ confidence interval, $H R$ hazard ratio, $N$ number of patients randomized in Asian subgroup, $Q 4 W$ every 4 weeks. $\mathrm{HR}<1$ favors denosumab 
Table 2 Treatment-emergent adverse events occurring in at least 20\% of patients in either treatment subgroup

\begin{tabular}{lcl}
\hline & $\begin{array}{l}\text { Denosumab 120 mg SC Q4W } \\
(\boldsymbol{N}=\mathbf{1 0 2})\end{array}$ & $\begin{array}{l}\text { Zoledronic acid 4 mg IV Q4W } \\
(\boldsymbol{N}=\mathbf{9 2})\end{array}$ \\
\hline Treatment-emergent AE, $n(\%)$ & $102(100.0)$ & $92(100.0)$ \\
Diarrhea & $52(51.0)$ & $47(51.1)$ \\
Nausea & $43(42.2)$ & $43(46.7)$ \\
Pyrexia & $39(38.2)$ & $38(41.3)$ \\
Upper respiratory tract & $38(37.3)$ & $37(40.2)$ \\
infection & & $29(31.5)$ \\
Constipation & $34(33.3)$ & $19(20.7)$ \\
Neutropenia & $32(31.4)$ & $31(33.7)$ \\
Decreased appetite & $31(30.4)$ & $28(30.4)$ \\
Insomnia & $30(29.4)$ & $20(21.7)$ \\
Cough & $28(27.5)$ & $20(21.7)$ \\
Anemia & $26(25.5)$ & $14(15.2)$ \\
Thrombocytopenia & $26(25.5)$ & $19(20.7)$ \\
Rash & $25(24.5)$ & $22(23.9)$ \\
Hypokalemia & $23(22.5)$ & $23(25.0)$ \\
Vomiting & $21(20.6)$ & $21(22.8)$ \\
Back pain & $19(18.6)$ & $19(20.7)$ \\
Neuropathy peripheral & $19(18.6)$ & $24(26.1)$ \\
Herpes zoster & $14(13.7)$ & \\
\hline P & &
\end{tabular}

Preferred terms are sorted by descending order of frequency in the denosumab group and coded using MedDRA version 19.0

$A E$ adverse event, $I V$ intravenously, MedDRA Medical Dictionary for Regulatory Activities, $Q 4 W$ every 4 weeks, $S C$ subcutaneously

respectively. Among the hypersensitivity AEs, rash (denosumab, 24.5\% [25/102]; zoledronic acid, 20.7\% [19/92]) was the most commonly reported hypersensitivity AE. Positively adjudicated atypical femur fracture was not reported in any patient.

\section{DISCUSSION}

In the current analysis, results from the Asian subgroup were overall comparable to results of the full study population [21]. Fewer Asian patients in the denosumab group developed a first on-study SRE versus those in the zoledronic acid group. The time to first on-study SRE had a trend favoring the denosumab group versus the zoledronic acid group. Moreover, no safety differences between denosumab and zoledronic acid were found in Asian patients with newly diagnosed MM.

A large-scale clinical trial comparing the effect of denosumab versus zoledronic acid on the time for SRE in newly diagnosed MM has been conducted [21]. Few previous reports have focused on denosumab versus zoledronic acid 
Table 3 Treatment-emergent adverse events potentially associated with renal toxicity

\begin{tabular}{lll}
\hline & $\begin{array}{l}\text { Denosumab } \mathbf{1 2 0} \mathbf{~ m g ~ S C} \mathbf{Q} \mathbf{4 W} \\
(\boldsymbol{N}=\mathbf{1 0 2})\end{array}$ & $\begin{array}{l}\text { Zoledronic acid 4 mg IV Q4W } \\
(\boldsymbol{N}=\mathbf{9 2})\end{array}$ \\
\hline $\begin{array}{l}\text { Treatment-emergent renal AE, } \\
n(\%)\end{array}$ & $9(8.8)$ & $20(21.7)$ \\
Blood creatinine increased & $5(4.9)$ & $12(13.0)$ \\
Renal failure & $2(2.0)$ & $1(1.1)$ \\
Urine output decreased & $2(2.0)$ & 0 \\
Acute kidney injury & 0 & $4(4.3)$ \\
Renal impairment & 0 & $3(3.3)$ \\
Blood urea increased & 0 & $1(1.1)$ \\
\hline
\end{tabular}

Acute renal failure standardized MedDRA query is used. Preferred terms are sorted by descending order of frequency in the denosumab group and coded using MedDRA version 19.0

$A E$ adverse event, $I V$ intravenously, MedDRA Medical Dictionary for Regulatory Activities, $Q 4 W$ every 4 weeks, $S C$ subcutaneously

for preventing SREs in Asian patients with MM. Similarly, in a retrospective study comparing treatment with denosumab to zoledronic acid in 242 Asian postmenopausal women with estrogen receptor-positive breast cancer, patients treated with denosumab had a significantly delayed time to the first on-study symptomatic skeletal event (SSE) and a decreased risk of SSEs [28].

In the exploratory endpoint of PFS, a trend towards improved PFS with denosumab versus zoledronic acid in the Asian subgroup was observed which was comparable with results in the full study population [21]. In the current subgroup analysis, most patients received frontline treatments with proteasome inhibitors and/or immunomodulatory drugs, allowing for unbiased comparisons of the SRE-protective effects of denosumab and zoledronic acid in the era of novel agents.

The incidences and types of AEs for the Asian subgroups were comparable to those in the full study population [21]. Denosumab has previously demonstrated a favorable safety profile in several clinical trials conducted in Asian populations for various indications, with the majority of AEs being mild in severity [29-31]. There was no significant difference in incidence of osteonecrosis of the jaw between the two groups. Nephrotoxicity is a well-known AE of zoledronic acid, and therefore renal toxicity is of particular concern when treating patients with renal impairment with zoledronic acid [32], which is not the case with denosumab $[17,20]$. As expected, in this Asian subgroup analysis, incidence of renal toxicity was higher in the zoledronic acid group than in the denosumab group (Table 3). Interestingly, a retrospective observational single-center study of 118 Japanese patients with bone metastases secondary to urological malignancies demonstrated a significant improvement in renal function among patients who switched from zoledronic acid to denosumab [33]. This observation may suggest a safer renal profile for denosumab than zoledronic acid as in the case of patients with MM.

Some limitations of this study include that no MM response data were collected and patients with a creatinine clearance of less than $30 \mathrm{~mL} / \mathrm{min}$ were not permitted to enroll because of the blinded nature of the study. It should be noted that a history of SRE prior to study enrollment is a risk factor for subsequent SREs, which may have confounded the time to on-study SRE between the denosumab and 
zoledronic acid groups. Additionally, differences in first-line therapy, specifically the higher rate of proteasome inhibitors with immunomodulatory drugs in the denosumab arm, may also have impacted on time to onstudy SRE and PFS. Lastly, it was a subgroup analysis with a limited number of patients that was insufficiently powered to detect a difference between the two treatment groups; therefore, the observed trends should be interpreted cautiously and may not be applicable to all Asian patients with MM and lytic bone lesions that are treated in clinical practice.

\section{CONCLUSIONS}

Results from the Asian subgroup analysis were comparable to those from the full study population. This analysis overall also supports the role of denosumab as an effective alternative treatment option to zoledronic acid in the standard of care for Asian patients with newly diagnosed MM and osteolytic lesions.

\section{ACKNOWLEDGEMENTS}

We thank the participants of the study.

Funding. This study was supported by Amgen Inc. and Amgen Global Publications. Sponsorship for the journal's Rapid Service and Open Access fee were funded by Amgen Inc.

Medical Writing Assistance. The authors thank Erin P. O'Keefe, PhD and Rick Davis, MS, RPh (Complete Healthcare Communications, a CHC Group company, North Wales, PA, USA), whose work was funded by Amgen Inc., and Yin C. Lin, PhD (Amgen Inc.), for medical writing assistance in the preparation of this manuscript.

Authorship. All named authors meet the International Committee of Medical Journal Editors (ICMJE) criteria for authorship for this article, take responsibility for the integrity of the work as a whole, and have given their approval for this version to be published.
Disclosures. Chang-Ki Min and Shang-Yi Huang have nothing to disclose. Sung-Soo Yoon has received consulting fees from or served an advisory role for Janssen, Takeda, Amgen, Celgene, Novartis, and Astellas; has received honoraria from Novartis; and has received research funding from Yuhan Pharmaceutical, Kyowa Kirin, Roche-Genentech. Kazuyuki Shimizu has received consulting fees from DaiichiSankyo Co., Ltd, and Fujimoto Pharmaceutical Group and is a member of the Denosumab 20090482 Global Steering Committee (Amgen Inc.). Wee Joo Chng has received research grants from Janssen, Merck, Celgene, and ASLAN and reports consulting fees from Amgen, AbbVie, Celgene, Janssen, Takeda, and Sanofi. Cheng-Shyong Chang has received consulting fees from Novartis, Roche, Takeda, Celgene, Janssen, AbbVie, and BMS and is on the speakers bureau for Novartis, Janssen, Roche, and BMS. Raymond Siu-Ming Wong has received research grants from Amgen, Bayer, Novartis, Archigen, Baxalta, Pfizer, Apellis, Roche, Boehringer Ingelheim, GlaxoSmithKline, and AbbVie; reports consulting fees from Amgen, Alexion, Boehringer Ingelheim, and GlaxoSmithKline; and is on the speakers bureau for Novartis, Amgen, Astellas, and Bayer. Seasea Gao and Steve Gordon are employees of Amgen Asia Holding Ltd and stockholders in Amgen Inc. Yang Wang and Anthony Glennane are employees of, and stockholders in, Amgen Inc.

Compliance with Ethics Guidelines. The study was conducted in accordance with Good Clinical Practice and study investigators obtained approval from their respective independent ethics committee or institutional review board before study initiation. Written informed consent was provided by patients before any protocol-specific procedures and before the administration of any study drug.

Data Availability. Qualified researchers may request data from Amgen clinical studies. Complete details are available at the following: http://www.amgen.com/datasharing.

Open Access. This article is licensed under a Creative Commons Attribution- 
NonCommercial 4.0 International License, which permits any non-commercial use, sharing, adaptation, distribution and reproduction in any medium or format, as long as you give appropriate credit to the original author(s) and the source, provide a link to the Creative Commons licence, and indicate if changes were made. The images or other third party material in this article are included in the article's Creative Commons licence, unless indicated otherwise in a credit line to the material. If material is not included in the article's Creative Commons licence and your intended use is not permitted by statutory regulation or exceeds the permitted use, you will need to obtain permission directly from the copyright holder. To view a copy of this licence, visit http:// creativecommons.org/licenses/by-nc/4.0/.

\section{REFERENCES}

1. Global Burden of Disease Cancer Collaboration, Fitzmaurice C, Abate D, et al. Global, regional, and national cancer incidence, mortality, years of life lost, years lived with disability, and disability-adjusted life-years for 29 cancer groups, 1990 to 2017: a systematic analysis for the Global Burden of Disease Study. JAMA Oncol. 2019;5:1749-68.

2. The Global Cancer Observatory. Malaysia fact sheet. http://gco.iarc.fr/today/data/factsheets/ populations/458-malaysia-fact-sheets.pdf. Accessed Dec 11, 2019.

3. The Global Cancer Observatory. Japan fact sheet. http:/gco.iarc.fr/today/data/factsheets/populations/ 392-japan-fact-sheets.pdf. Accessed Dec 11, 2019.

4. The Global Cancer Observatory. Republic of Korea fact sheet. http:/gco.iarc.fr/today/data/factsheets/ populations/410-korea-republic-of-fact-sheets.pdf. Accessed Dec 11, 2019.

5. The Global Cancer Observatory. Singapore fact sheet. http://gco.iarc.fr/today/data/factsheets/ populations/702-singapore-fact-sheets.pdf. Accessed Dec 11, 2019.

6. The Global Cancer Observatory. Asia Fact Sheet. http:/gco.iarc.fr/today/data/factsheets/populations/ 935-asia-fact-sheets.pdf. Accessed Dec 11, 2019.

7. Liu J, Liu W, Mi L, et al. Incidence and mortality of multiple myeloma in China, 2006-2016: an analysis of the Global Burden of Disease Study 2016. J Hematol Oncol. 2019;12:136.

8. Tang CH, Liu HY, Hou HA, et al. Epidemiology of multiple myeloma in Taiwan, a population based study. Cancer Epidemiol. 2018;55:136-41.

9. O'Donnell EK, Raje NS. Myeloma bone disease: pathogenesis and treatment. Clin Adv Hematol Oncol. 2017;15:285-95.

10. Roodman GD. Mechanisms of bone metastasis. N Engl J Med. 2004;350:1655-64.

11. Lipton A, Fizazi K, Stopeck AT, et al. Superiority of denosumab to zoledronic acid for prevention of skeletal-related events: a combined analysis of 3 pivotal, randomised, phase 3 trials. Eur J Cancer. 2012;48:3082-92.

12. Terpos E, Szydlo R, Apperley JF, et al. Soluble receptor activator of nuclear factor kappaB ligandosteoprotegerin ratio predicts survival in multiple myeloma: proposal for a novel prognostic index. Blood. 2003;102:1064-9.

13. Terpos E, Ntanasis-Stathopoulos I, Gavriatopoulou M, Dimopoulos MA. Pathogenesis of bone disease in multiple myeloma: from bench to bedside. Blood Cancer J. 2018;8:7.

14. Kim K, Lee JH, Kim JS, et al. Clinical profiles of multiple myeloma in Asia-an Asian Myeloma Network study. Am J Hematol. 2014;89:751-6.

15. National Cancer Institute. SEER cancer stat facts: myeloma. https://seer.cancer.gov/statfacts/html/ mulmy.html. Accessed Dec 12, 2019.

16. Anderson K, Ismaila N, Flynn PJ, et al. Role of bonemodifying agents in multiple myeloma: American Society of Clinical Oncology clinical practice guideline update. J Clin Oncol. 2018;36:812-8.

17. Kanellias N, Gavriatopoulou M, Terpos E, Dimopoulos MA. Management of multiple myeloma bone disease: impact of treatment on renal function. Expert Rev Hematol. 2018;11:881-8.

18. Lee OL, Horvath N, Lee C, et al. Bisphosphonate guidelines for treatment and prevention of myeloma bone disease. Intern Med J. 2017;47:938-51.

19. Mhaskar R, Kumar A, Miladinovic B, Djulbegovic B. Bisphosphonates in multiple myeloma: an updated network meta-analysis. Cochrane Database Syst Rev. 2017;12:CD003188.

20. Terpos E, Sezer O, Croucher PI, et al. The use of bisphosphonates in multiple myeloma: recommendations of an expert panel on behalf of the 
European Myeloma Network. Ann Oncol. 2009;20: 1303-17.

21. Raje N, Terpos E, Willenbacher W, et al. Denosumab versus zoledronic acid in bone disease treatment of newly diagnosed multiple myeloma: an international, double-blind, double-dummy, randomised, controlled, phase 3 study. Lancet Oncol. 2018;19:370-81.

22. Henry DH, Costa L, Goldwasser F, et al. Randomized, double-blind study of denosumab versus zoledronic acid in the treatment of bone metastases in patients with advanced cancer (excluding breast and prostate cancer) or multiple myeloma. J Clin Oncol. 2011;29:1125-32.

23. Amgen Inc. Xgeva (denosumab) prescribing information. https://www.pi.amgen.com/ /media/ amgen/repositorysites/pi-amgen-com/xgeva/xgeva pi.pdf. Accessed Nov 14, 2019.

24. Henry D, Vadhan-Raj S, Hirsh V, et al. Delaying skeletal-related events in a randomized phase 3 study of denosumab versus zoledronic acid in patients with advanced cancer: an analysis of data from patients with solid tumors. Support Care Cancer. 2014;22:679-87.

25. Stopeck AT, Lipton A, Body JJ, et al. Denosumab compared with zoledronic acid for the treatment of bone metastases in patients with advanced breast cancer: a randomized, double-blind study. J Clin Oncol. 2010;28:5132-9.

26. Fizazi K, Carducci M, Smith M, et al. Denosumab versus zoledronic acid for treatment of bone metastases in men with castration-resistant prostate cancer: a randomised, double-blind study. Lancet. 2011;377:813-22.

27. Smith MR, Coleman RE, Klotz L, et al. Denosumab for the prevention of skeletal complications in metastatic castration-resistant prostate cancer: comparison of skeletal-related events and symptomatic skeletal events. Ann Oncol. 2015;26: 368-74.

28. Zhang C, Zhang F, Liang G, et al. Denosumab versus zoledronic acid for preventing symptomatic skeletal events in Asian postmenopausal women with oestrogen-receptor-positive advanced breast cancer: an outcome analyses with a mean follow-up of 3 years. BMC Musculoskelet Disord. 2018;19:424.

29. Koh JM, Chung DJ, Chung YS, et al. Assessment of denosumab in Korean postmenopausal women with osteoporosis: randomized, double-blind, placebo-controlled trial with open-label extension. Yonsei Med J. 2016;57:905-14.

30. Nakamura T, Matsumoto T, Sugimoto T, et al. Clinical Trials Express: fracture risk reduction with denosumab in Japanese postmenopausal women and men with osteoporosis: denosumab fracture intervention randomized placebo controlled trial (DIRECT). J Clin Endocrinol Metab. 2014;99: 2599-607.

31. Takeuchi $\mathrm{T}$, Tanaka $\mathrm{Y}$, Ishiguro $\mathrm{N}$, et al. Effect of denosumab on Japanese patients with rheumatoid arthritis: a dose-response study of AMG 162 (Denosumab) in patients with RheumatoId arthritis on methotrexate to Validate inhibitory effect on bone Erosion (DRIVE)-a 12-month, multicentre, randomised, double-blind, placebo-controlled, phase II clinical trial. Ann Rheum Dis. 2016;75: 983-90.

32. Zometa ${ }^{\circledR}$ (zoledronic acid). Full prescribing information, Novartis Pharmaceuticals Corporation, East Hanover, NJ, 2016.

33. Yamasaki M, Yuasa T, Uehara S, et al. Improvement of renal function by changing the bone-modifying agent from zoledronic acid to denosumab. Int J Clin Oncol. 2016;21:1191-5. 